$\Rightarrow$

\title{
A trip down memory lane
}

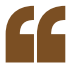 \\ parental \\ responses to \\ the environ- \\ ment are \\ passed to \\ offspring by \\ small RNAs}

Studies in model organisms have suggested that behaviour can be passed down through generations by transgenerational epigenetic inheritance (TEI), but the underlying mechanisms are unclear. Using Caenorhabditis elegans, two groups now find that parental responses to the environment are passed to offspring by small RNAs. Moore et al. show that worms transmit 'avoidance behaviour' for four generations via the Piwi-associated RNA (piRNA) prg-1. Posner et al. show that the worms' nervous system transmits 'chemotactic behaviour' for three generations via the RNA interference (RNAi)deficient 4 (RDE-4)-dependent generation of neuronal endogenous small interfering RNAs (endo-siRNAs).

C. elegans are more attracted to pathogenic Pseudomonas aeruginosa (PA14) than to a non-pathogenic laboratory Escherichia coli strain (OP50); however, they quickly learn to avoid PA14. Moore et al. observed that mothers exposed to PA14 for 24 hours developed avoidance behaviour that was passed to the next four generations, indicating that this behaviour is subject to TEI.

Notably, compared to their OP50-exposed counterparts, mothers exposed to PA14 and their F1 progeny showed a marked upregulation of genes associated with adult neurons. Focusing on the neuronal gene daf- 7 , which encodes a TGF- $\beta$ ligand, the authors found that PA14 increased

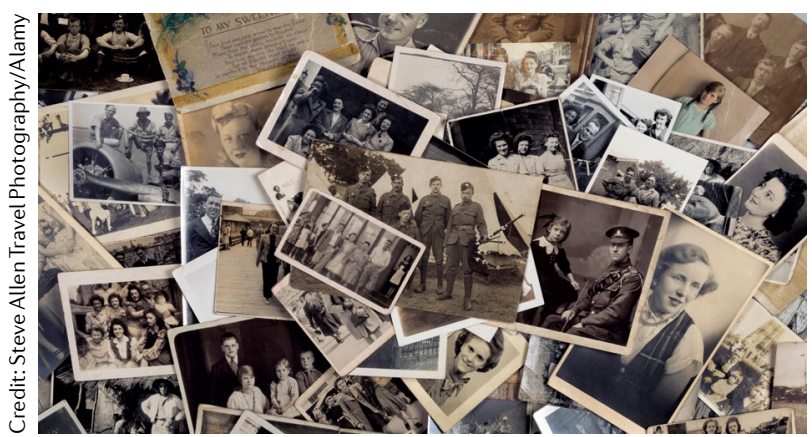

daf-7 expression in ASI sensory neurons for four generations. Worms lacking ASI neurons or daf-7 avoided PA14 but could not pass this behaviour down, and depletion of daf-7 in the F1 progeny of PA14-treated mothers abolished avoidance. These data suggest that daf-7 in the ASI mediates the TEI of avoidance behaviour and that it acts in the F1 generation.

Small RNAs have been implicated in TEI, and the authors observed that piRNAs were differentially expressed in PA14-treated mothers compared with controls. Interestingly, mothers lacking the piRNA prg-1 learnt avoidance but did not pass this behaviour to their progeny, in which daf-7 expression was not upregulated in ASI neurons. Thus, prg-1 is required for the upregulation of daf-7 in ASI neurons.

Downstream of prg-1, RNA-dependent RNA polymerases (RDRPs) amplify primary siRNAs and trigger the production of heritable endo-siRNAs; endo-siRNAs bind to germline Argonaute proteins, such as heritable RNAi-deficient 1 (HRDE-1), to execute TEI. Worms mutant for RDRPs learnt avoidance behaviour but did not pass it to their progeny, suggesting that prg-1 and its downstream RNAi pathway confer the TEI of avoidance behaviour.

Finally, as the progeny of PA14exposed mothers (which had a survival advantage) also avoided non-pathogenic Pseudomonas, avoidance behaviour may be lost in the fifth generation to re-attract worms to nutritious Pseudomonas.

Posner et al. hypothesized that neuronal endo-siRNAs dependent on RDE-4 (a double-stranded RNA-binding protein) for their production might produce a heritable response. They took $r d e-4$ mutant worms with an RNAi-deficient phenotype and rescued RDE-4 expression in neurons (RDE-4 rescue worms); 476 small RNAs targeting a specific gene ('STGs') were upregulated in RDE-4 rescue worms compared with $r d e-4$ mutants. By isolating neurons from these worms, the authors found that 46 RDE-4dependent STGs were specific to neurons (neuronal STGs).

Sequencing small RNAs from worm gonads identified 1,287 germline STGs that were affected by the neuronal expression of RDE-4, suggesting that changes in neuronal STGs are communicated between generations. Whole-worm sequencing revealed that 189 STGs seemed to be inherited by three generations of worms, as were changes in the level of five $m R N A s$ regulated by germline STGs, including of the foraging gene saeg-2. The inherited STGs were enriched for those bound by HRDE-1, in line with the role of this protein in TEI. Thus, neuronal RDE-4 may enable the TEI of HRDE-1-bound endo-siRNAs and of the regulation of their target genes.

In search of a role for RDE-4mediated TEI, the authors observed that $r d e-4$ mutants showed defective chemotaxis upon heat stress. This defect was partially rescued by the neuronal-specific expression of $r d e-4$ in a manner dependent on hrde-1. Furthermore, chemotactic behaviour improved in $\mathrm{F} 3$ progeny null for $r d e-4$ when they descended from $r d e-4^{+/-}$or RDE-4 rescue worm great-grandparents. Thus, the neuronal expression of RDE-4 affects chemotaxis across generations. Further experiments suggested that RDE-4 partially restores chemotaxis by silencing saeg-2, which underwent TEI and is highly expressed in the germline in the absence of RDE-4.

In sum, these studies show how, in worms, TEI can communicate neuronal processes.

Katharine H. Wrighton ORIGINAL ARTICLE Moore, R. S. et al. Piwi/PRG-1 argonaute and TGF- $\beta$ mediate transgenerational learned pathogenic avoidance. Cell 177, 1827-1841 (2019)| Posner, R. et al. Neuronal small RNAs control behavior transgenerationally. Cell 177, 1814-1826 (2019) 